\title{
Laser Ultrasonics System for Measurement of Speed of Sound in Gases
}

\author{
J. M. S. Sakamoto*, , G. M. Pacheco ${ }^{\dagger}$, C. Kitano** and H. A. Machado ${ }^{\ddagger}$ \\ *Division of Photonics, Instituto de Estudos Avançados - IEAv, São José dos Campos, SP 12228-001, Brazil \\ ${ }^{\dagger}$ Department of Microwave and Optoelectronics, Instituto Tecnológico de Aeronáutica - ITA, São José dos \\ Campos, SP 12228-900, Brazil \\ ${ }^{* *}$ Department of Electrical Engineering, Universidade Estadual Paulista - UNESP, Ilha Solteira, SP 15385-000, \\ Brazil \\ ${ }^{\ddagger}$ Division of Space Systems, Instituto de Aeronáutica e Espaço - IAE, São José dos Campos, SP 12228-900, \\ Brazil
}

\begin{abstract}
In this work we developed a setup to measure the speed of sound in gases using a laser ultrasonics system. The mentioned setup is an all optical system composed by a Q-switched Nd:YAG laser to generate the sound waves, and a fiber optical microphone to detect them. The Nd:YAG provided a laser pulse of approximately $420 \mathrm{~mJ}$ energy and $9 \mathrm{~ns}$ of pulse width, at the wavelength of $1064 \mathrm{~nm}$. The pulsed laser beam, focused by a positive lens, was used to generate an electrical breakdown (in the gas) which, in turn, generates an sound wave that traveled through a determined distance and reached the fiber optical microphone. The resulting signal was acquired in an oscilloscope and the time difference between the optical pulse and the arrival of the sound waves was used to calculate the speed of sound, since the distance was known. The system was initially tested to measure the speed of sound in air, at room pressure and temperature and it presented results in agreement with the theory, showing to be suitable to measure the speed of sound in gases.
\end{abstract}

Keywords: Laser ultrasonics, Speed of sound, Fiber optical microphone. PACS: 43.35.Ae, 78.20.hc, 43.20.Ye.

\section{INTRODUCTION}

The determination of thermodynamic properties of gases has great technological importance in scientific and industrial applications, as the fabrication of integrated circuits, production of composite material and, in aerospace area, the characterization of the products from the combustion of propulsive systems. In the case of the aerospace applications, the measurement of thermodynamic properties of gases expelled by jet or rocket engines is particularly difficult and, in general, theoretical models and computational simulation are used for the evaluation. However, experimental results are required for the validation of the acuity of these models [1]. Within this context, the measurement of the speed of sound in a gas flow provided directly from a rocket engine test ("bank shot") would be of great interest since it would allow to obtain these properties, and consequently, more accurate information on these gases, the resulting heat flow and eventually the actual performance of the engine.

As a first step to achieve a measurement of the speed of sound in a gas flow, we present in this work an experimental setup to measure the speed of sound in gases initially in the static condition, at room pressure and temperature (RPT). This setup works based on the laser ultrasonics technique: a high energy pulsed laser is used to generate acoustic waves and a fiber optical microphone is used to detect it. Usually, an optical interferometer (as a Michelson, Mach-Zehnder, Fabry-Perot, or photorefractive) is used to detect the ultrasonic waves. Another type of optical detector that could be used for the measurement of speed of sound is the laser-induced thermal acoustics (LITA) [2, 3]. However, the fiber optical microphone presents as advantages the use of inexpensive optical components and it is ease to assembly and align.

\section{EXPERIMENTAL SETUP}

The experimental setup developed in this work is shown in Fig. 1 (a). This setup counts with a Q-switched Nd:YAG laser which has the following characteristics: wavelength of $1064 \mathrm{~nm}$, pulse width (FWHM) of $9 \mathrm{~ns}$, maximum energy per pulse of $420 \mathrm{~mJ}$, and maximum repetition rate of $10 \mathrm{~Hz}$. This laser, focused by a positive lens (focal length of 100 


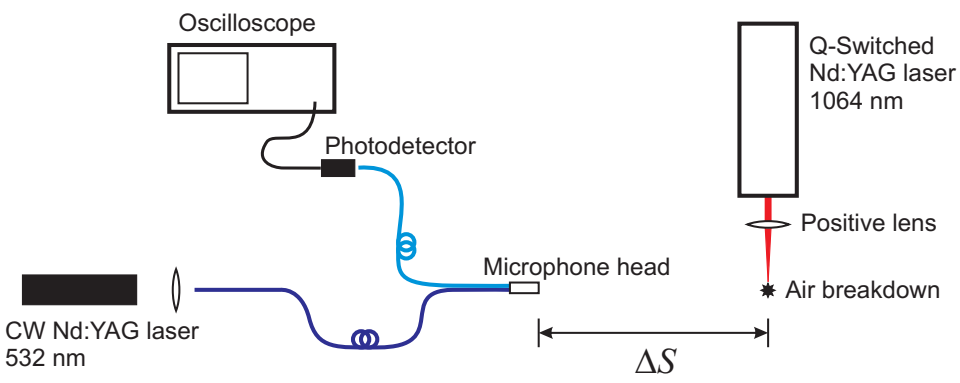

(a)

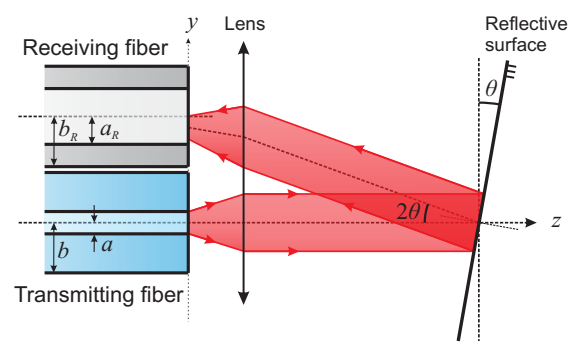

(b)

FIGURE 1. Experimental setup. (a) Laser ultrasonics system. (b) Microphone head detail.

$\mathrm{mm}$ ), is used to generate an electrical breakdown (shown in Fig. 2) in the dielectric of the gas (air in this case) which in turn generates an acoustic pulse. This pulse travels a determined distance, reaches the fiber optical microphone and the detected signal is registered in an oscilloscope. The light emitted by the air breakdown is used as trigger, detected directly by the photodetector. The time difference between the trigger and the acoustic pulse is used to calculate the speed of sound.

The fiber optical microphone is composed by two optical fibers, a positive lens and a diaphragm (reflective surface), as shown in Fig 1 (b). One fiber, called transmitting fiber (core radius $a=4 \mu \mathrm{m}$, cladding radius $b=62.5 \mu \mathrm{m}$, and numerical aperture $N A=0.12$ ), is used to carry the light from an optical source (in this case a CW Nd:YAG laser at $532 \mathrm{~nm}$ and $100 \mathrm{~mW}$ of power). The other, called receiving fiber (with the same parameters as the transmitting fiber, i.e., $a_{R}=a=4 \mu \mathrm{m}$ and $b_{R}=b=62.5 \mu \mathrm{m}$ ), is used to collect the light reflected by the diaphragm and carry it to a photodetector. The fibers are aligned side-by-side and fixed together keeping their tips aligned too. A positive lens (focal distance of $7.4 \mathrm{~mm}$ ) is positioned in front of the fibers to play two main roles: collimate the light from the transmitting fiber and focus the light reflected by the diaphragm. If the diaphragm is tilted by an angle of $\theta$, the total deviation is $2 \theta$. This angle variation causes the optical spot to travel on the core of the receiving fiber, modulating the amount of light coupled to it. Summarizing, the fiber optical microphone converts acoustic waves into light intensity variation which in turn is converted to voltage variation by a photodetector transimpedance circuit.

The characteristic curve of the microphone used in this work presents a region with a positive inclination slope and after a peak, a negative inclination slope as shown in Fig. 3. In this figure, the $x$ axis is the diaphragm's angle, $\theta$, and the $y$ axis is the normalized output, $\eta$. To work as a microphone and detect acoustic waves, one can use the characteristic curve to apply an angular bias on the microphone diaphragm, rotating it. This is accomplished with a rotation stage in which the diaphragm is placed. The rotation stage is turned to find the peak of the curve. At this point, the output voltage is called $V_{\max }$. After that, the translation stage is moved until it reaches $0.5 V_{\max }$ or $0.6 V_{\max }$, which corresponds to the bias $\left(\theta_{B I A S}\right)$ necessary to the microphone convert the acoustic waves in light variation (regarded as the quiescent point), in a linear fashion. In this measurement we used the positive slope, but the negative slope could also be used instead. Around the bias point, small variations of the diaphragm's angle are converted to variation of the

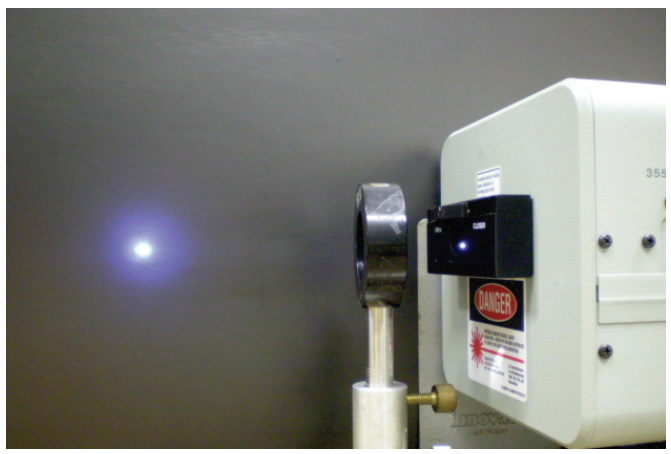

FIGURE 2. Electrical breakdown generated by the laser. 


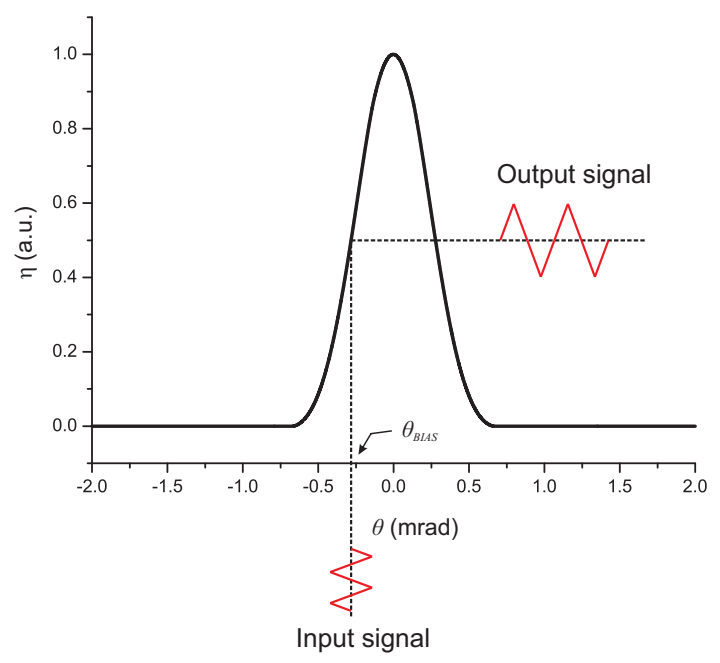

FIGURE 3. Characteristic curve of the microphone.

light intensity viewed by the photodetector [4]. The photodetector is part of the transimpedance circuit, designed to detect frequencies up to $85 \mathrm{MHz}$. Due to this configuration, the fiber optical microphone is capable to detect acoustic waves in gases.

\section{RESULTS}

The Nd:YAG laser was turned on with repetition rate of $5 \mathrm{~Hz}$ in order to average and acquire the waveforms on the oscilloscope. The typical waveform presents the optical pulse at $t_{o}=0 \mathrm{~ms}$ and the arrival of the acoustic pulse (first peak) at a time $t$, whose time difference $\left(\Delta t=t-t_{o}\right)$ is measured, as shown in Fig. 4. Using six different distances, $\Delta S$, the six corresponding time differences, $\Delta t$, were acquired. The speed of sound was then calculated and averaged. The results are shown in Table 1 . The measurements were accomplished at the temperature of approximately $293 \mathrm{~K}$ $\left(20{ }^{\circ} \mathrm{C}\right)$.

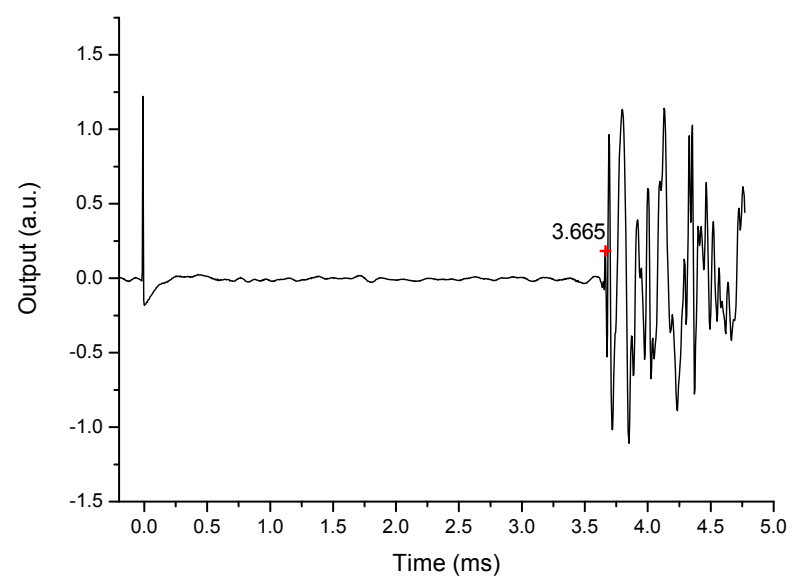

FIGURE 4. Detected waveform $(\Delta S=125.8 \mathrm{~cm})$. 
TABLE 1. Speed of sound measurements.

\begin{tabular}{llll}
\hline & $\begin{array}{l}\Delta S[\mathbf{m}] \\
\times 10^{-2}\end{array}$ & $\begin{array}{l}\Delta t[\mathbf{s}] \\
\times 10^{-3}\end{array}$ & $v[\mathbf{m} / \mathbf{s}]$ \\
\hline 1 & 136.3 & 3.980 & 342.7 \\
2 & 125.8 & 3.665 & 343.2 \\
3 & 113.3 & 3.305 & 342.8 \\
4 & 104.3 & 3.065 & 340.3 \\
5 & 94.3 & 2.750 & 342.9 \\
6 & 84.4 & 2.470 & 341.7 \\
\hline Mean & - & - & 342.3 \\
Std. dev. & - & - & 1.1 \\
\hline
\end{tabular}

According to the Table 1, the mean value of the speed of sound is $342.3 \mathrm{~m} / \mathrm{s}$ with standard deviation of $1.1 \mathrm{~m} / \mathrm{s}$, i.e., precision of $0.3 \%$. The theoretical speed of sound as a function of temperature is given by [5]:

$$
v=331.4+0.607 T[\mathrm{~m} / \mathrm{s}]
$$

where $T$ is the temperature in ${ }^{\circ} \mathrm{C}$. Using $T=293 \mathrm{~K}$ and equation (1), the speed of sound is $v=343.5 \mathrm{~m} / \mathrm{s}$ which means that the experimental value $(342.3 \mathrm{~m} / \mathrm{s})$ presents a relative error of $0.4 \%$ in comparison with the theoretical value.

\section{CONCLUSIONS}

In conclusion, the presented setup was suitable for the measurement of speed of sound in the static condition, presenting an experimental result in agreement with the theory, with a relative error of $0.4 \%$ and precision of $0.3 \%$. The experimental setup proposed has advantages over the optical interferometer or the LITA due to its simplicity and low cost. Based on this outcome, the authors intend to adjust the measurement system to accomplish the evaluation in dynamic and more severe condition, at high pressure and temperature.

\section{ACKNOWLEDGMENTS}

The authors wish thank Dr. Rogério M. Cazo for the support on the design and manufacturing of the electronic circuits, Dr. Nicolau A. S. Rodrigues, Dr. Rudimar Riva and Tales B. Caldas for the discussions about lasers and the optical design. One of the authors (JMSS) acknowledges the Brazilian sponsor agencies CNPq (for the provision of a scholarship under the process number 142191/2007-8) and CAPES (for the provision of an international scholarship under the process number 4697/08-1).

\section{REFERENCES}

1. S. Lago, and P. A. G. Albo, The Journal of Chemical Thermodynamics 41, 506-512 (2009), ISSN 0021-9614.

2. R. C. Hart, R. J. Balla, and G. C. Herring, Applied Optics 38, 577-584 (1999).

3. R. C. Hart, R. J. Balla, and G. C. Herring, J. Acoust. Soc. Am. 108, 1946-1948 (2000).

4. J. M. S. Sakamoto, and G. M. Pacheco, Physics Procedia 3, 651-658 (2010), in International Congress on Ultrasonics, Santiago de Chile, January 2009.

5. J. Eargle, The Microphone Workbook, Focal Press, Burlington, 2004, pp. 7-21. 
Copyright of AIP Conference Proceedings is the property of American Institute of Physics and its content may not be copied or emailed to multiple sites or posted to a listserv without the copyright holder's express written permission. However, users may print, download, or email articles for individual use. 